Original Research

\title{
Theoretical Modelling of Liquid Crystal Electro-Optical Material Display Elements to Achieve Optimal Low Power Consumption Ship Stealth
}

\author{
Christopher R. Lavers ${ }^{1,2, *}$
}

1. Dartmouth Centre for Sea Power and Strategy, Plymouth University at Britannia Royal Naval College, Dartmouth, TQ6 OHJ, UK; E-Mail: christopher.lavers@plymouth.ac.uk

2. Visiting Research Fellow, CCW Pembroke College, Oxford OX1 1DW, UK.

* Correspondence: Christopher R. Lavers; E-Mail: christopher.lavers@plymouth.ac.uk

Academic Editor: Hossein Hosseinkhani

Recent Progress in Materials

2021, volume 3, issue 2

doi:10.21926/rpm.2102019
Received: December 29, 2020

Accepted: April 23, 2021

Published: May 14, 2021

\begin{abstract}
Advanced optical stealth techniques are of growing importance in the role of manned and unmanned aircraft or ship platforms. We consider here the concept of using passive reflective display elements rather than high power active light projection to achieve a low observable camouflaged ship platform. This paper presents practical consideration towards minimal radiated power user-driven requirements using adaptive liquid crystal electrooptical methods. Optical reflectivity for a multi-layer liquid crystal cell structure composed of a glass/ ITO/ aligning layer/ liquid crystal/ aligning layer/ ITO/ glass will support optical guided modes. Reflectivity as a function of angle and voltage are compared with theory generated from a Fresnel matrix formalism. Voltage device modulated reflectivities are then simulated across a ship platform to evaluate this method for low power consumption, with minimal radiated optical radiation.
\end{abstract}

\section{Keywords}

Optical guided modes, environmental sensing, optical modelling

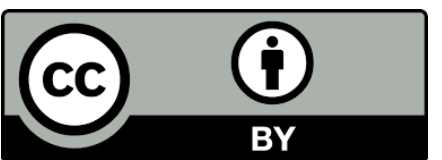

(C) 2021 by the author. This is an open access article distributed under the conditions of the Creative Commons by Attribution License, which permits unrestricted use, distribution, and reproduction in any medium or format, provided the original work is correctly cited. 


\section{Introduction}

Optical or optimal stealth technology today is seen as essential to the role of manned and unmanned aircraft, with active projection or passive reflective display elements. In the same way optical methods may help to reduce the optical signature of a small stealth warship platform like the Swedish Visby stealth corvette following the method demonstrated recently by Lavers and Johnson [1], although proposed seven years ago [2]. Optical prism-coupling is an established method used to probe advanced electro-optical materials such as Liquid Crystal (LC) layers, to provide vital optical information on material parameters, and is a potential means to providing large switchable display surface areas. Leaky cells fabricated from consecutive multiple layers of: glass/ ITO/ alignment layer/ LC/alignment layer/ ITO/ glass will support propagating optical guided modes. Reflectivity as a function of both incident angle and applied voltage are compared with theory generated from Fresnel optical theory. Real achievable reflectivities may be used to simulate contrast across a ship's platform with typically up to 60,000 pixel elements, to evaluate methods for minimising passive power consumption.

\subsection{History of Optical Stealth}

Stealth, or Low Observable Technology (LOT), is concerned with making any platform: surface combatant warship, Unmanned Aerial Vehicle (UAV), or manned aircraft, less visible to Radar, Infra-Red or other detection methods. During World War One, Britain was losing many warships, so the Royal Navy, desperate for a solution, attempted to hide them though bizarre dazzle camouflage paints schemes of colourful and abstract cubist blocks and stripes, so vessels would 'blur' into a complex background of sky, sea, and coastline. Normal Wilkinson CBE, primarily maritime painter, was the first artist credited with the use disruptive naval camouflage patterns. Dazzle or 'razzle-dazzle' camouflage was used widely at the end of World War One and to a lesser extent in World War Two, figure 1, which shows British HMS Uranus and HMS Jervis engaged in the Normandy landings. Detection whether it is visual, radar, or infra-red (heat) is largely about contrast. Clothing, airframes, ships or vehicles matching the background land or littoral environment forms the basis of concealment. Addition of various coloured patterns makes it less likely to be detected in a complex environmental and forms the basis of disruptive camouflage. The German Air Force (1913) was the first to try to make aircraft invisible with a transparent monoplane of light colours, detectable at a height of $900 \mathrm{ft}$. Today, such invisibly cloaked maritime stealth aircraft may thus also be hidden flying over their enemies, safely undetected.

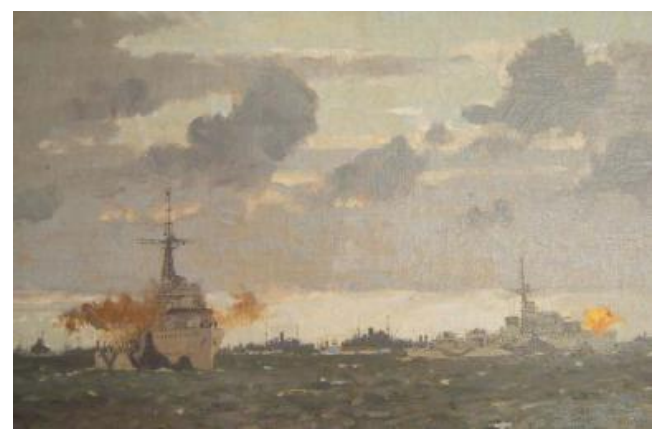

Figure 1 The Normandy landings of 6 June 1944 HMS Uranus and HMS Jervis, in the early morning with landing craft waiting to go in, painting by Norman Wilkinson, held at Britannia Royal Naval College, Dartmouth (C) CR Lavers. 
Current maritime dazzle camouflage is entirely passive, and can only be changed by painting the platform. Active methods alter appearance in near real time to confuse enemies. Active or adaptive camouflage uses emerging technologies which blend objects into their surroundings with panels or coatings to alter colour, luminance or reflectivity. Active camouflage provides concealment from visual detection. One proven practical military application of this concept was during World War Two in efforts to defeat the U-boat menace. Allied aircraft trying to target surfaced submarines had a significant problem because German submarine lookouts could spot the dark silhouettes of incoming aircraft a long distance away, diving to the safety of deeper waters. By 1940 US researchers made aircraft effectively 'invisible', adjusting the brightness of lights on leading wing edges to hide them. Project Yehudi's Avenger bombers suitably camouflaged were able to reduce their own platform detection down to about 2 miles. In the same manner the prototype F-117A used distributed optical fibre lighting on its lower wing surfaces to minimise optical contrast against the background sky [3], now optimised with the X47B Pegasus UAV (figure 2), recording sky and using lights below to blur outline, reducing overall contrast. This military detection phenomenon is often termed isoluminance, which arises when the eye detects equal brightness from both a target and its background, making recognition of the target difficult, and ideally impossible to the human eye, which cannot distinguish the outline of the platform for it to be perceived.

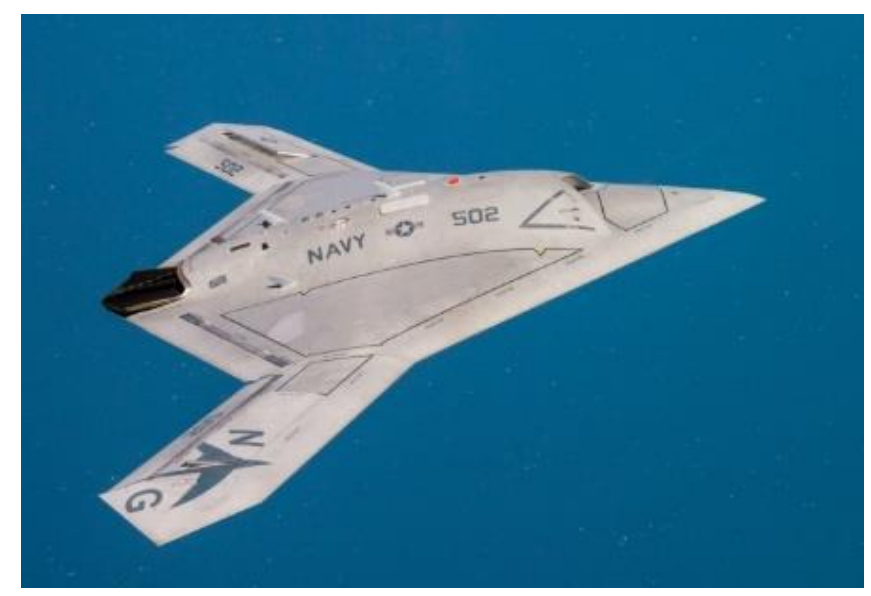

Figure 2 X47B Pegasus.

However, projection requires high power levels, and power consumption reduces endurance and other important user platform design factors. The importance of endurance (106 responses), and power consumption (41 responses) from military and civilian UAV communities, from a userled upstream/downstream UAV sensors market project [1]. It is critical to develop platforms around key customer requirements, appreciating limitations which include: power consumption and endurance, see figure 3 . Without evaluation of what military customer require, there cannot be a proper technical evaluation. 


\section{Power Consumption}

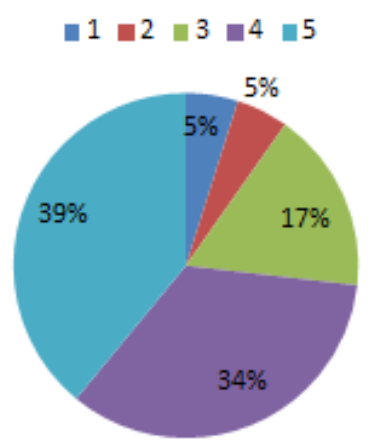

\section{Endurance}

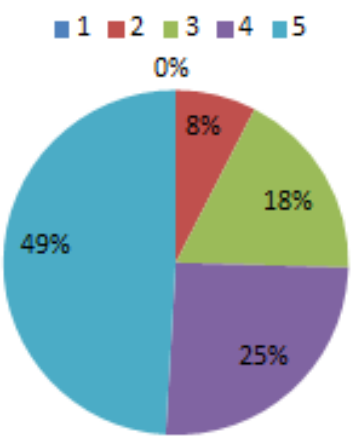

Figure 3 Unmanned Aerial Vehicle platform military and civilian questionnaire responses, using a Likert scale from 1 to 5 , where 1 is not at all important, to 5 very important.

\section{Theoretical Approach}

Conformable optical reflectivity element design uses Fresnel theory for multi-layer modelling of liquid crystals. Liquid crystal cell model design allows optimal cell reflectivity changes to simulated real reflectivity across proposed ship or UAV platform surfaces. A FORTRAN scattering matrix method [4] accounts for reflection / transmission coefficients at media interfaces, coupling incoming fields with a stable matrix for data fitting (figure 4). Our method calculates reflectivity as a function of incident angle for multi-layer media as a series of isotropic slabs of thickness below a wavelength, but can accommodate uniaxial layers for electro-optical materials such as ferroelectric liquid crystals. This scattering method [4] is an inherently more stable approach than traditional transfer matrix methods developed by Berreman and Scheffer, and Azzam and Bashara [5, 6] (figure 4). As illustrated in figure 4, the forward and backward travelling electric field vectors in media 1 at the interface are related to the forward and backward travelling electric field vectors in medium 2. Writing all these together in matrix form, generates what is known as the scattering matrix S.

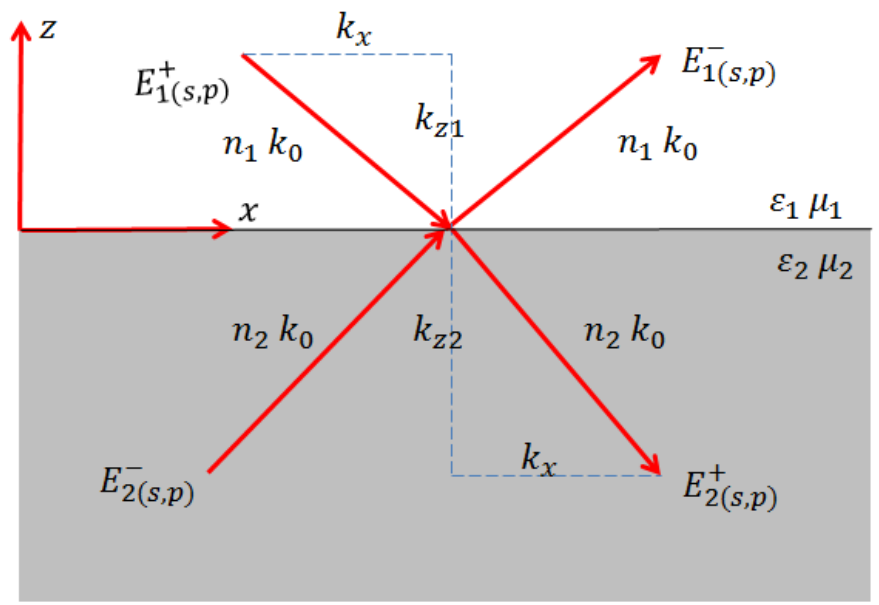

Figure 4 Scattering matrix approach. 
We employ this methodology to simulate the level of optical manipulation possible using liquid crystal materials as the electro-optical element. The simple approach of altering platform reflectivity in response to ambient environmental conditions is hardly new, but the low power advantages of adjusting ambient reflection levels is attractive to the military. Accurately modelled liquid crystal cell voltage dependent reflectivity provides the ability to control large number of pixels optically in near real-time. Non-optical active systems exist, e.g. BAE recently demonstrated an active thermal technique now in production [7], besides an adaptive thermal camouflage using graphene [8], and even an adaptive infra-red reflectivity system inspired by cephalopods [9], whilst other users have demonstrated this principle in the NIR spectra. Several active camouflage designs are currently under development, based upon that started with the Lockheed Have Blue Stealth prototype in the 1970s [3] which had active light ports on the bottom surface. These light ports were connected to fibre optic lines which were modulated by a detector on the opposite side of the aircraft.

Depending on the altitude of the aircraft, colour and brightness of the environmental background, visual detection may be reduced. However, the first serious attempt to develop maritime active stealth was in 2002 when Global Atlantic Inc., in collaboration with Olins Smart Boat Corporation, and Boston Whaler attempted to produce a working, active camouflage system for a small multi-purpose vessel [10]. Their system used OLEDs in conjunction with a small 360 degree camera to display images of the vessel's surrounding onto its surface, following Moynihan and Langevin [11] who purposed lightweight optoelectronics systems that use image sensors and display panels, to 'cloak' an object $10 \mathrm{~m}$ long, by $3 \mathrm{~m}$ high, by $5 \mathrm{~m}$ wide, weighing less than $45 \mathrm{~kg}$. Limited optical camouflage using retroreflective projection has been demonstrated [12]. However, the requirement for a retroreflective layer behind any cloaked object and projection camera currently is not practical. The UK, and NASA, are also working on active camouflage, as are Canadian and German military researchers with a chameleon-like armoured vehicle [13], all including a network of electronic flat-panel displays with cameras.

Instead, liquid crystal technology lends itself to flexible displays. Reflective LCDs offer longer battery lifetimes than transmission-based LCD systems, because no extra illumination is needed, relying on ambient light, but may suffer from reduced contrast. Several companies are working on cholesteric LCD technology (Kent display, $\mathrm{OH}$ ) and nematic (LCDs relying on bistability normally associated with SC* phase ferroelectric LCDs, with Zenithal Bistable Displays (ZBD). Active camouflage is also underway in aviation with voltage-driven FLC display panels of thin films that change colour when an electric current is passed through them, but require a lot of energy. The same principle is examined by Yablonovitch [14] in a report on electrochromic adaptive infra-red camouflage, fabricating the first all polymer electrochromic cell, achieving $80 \%$ contrast and switching in $67 \mathrm{~ms}$, essentially at video rates.

We demonstrate how to minimise the optical reflectivity of a Visby stealth corvette in the public domain, (figure 5) used in varied littoral environment roles relevant to the maritime operations of the Royal Swedish Navy. 


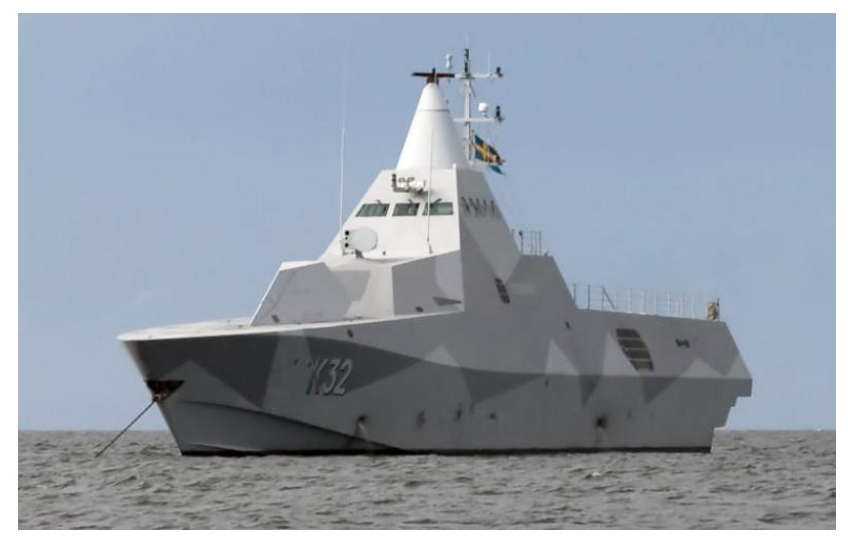

Figure 5 Visby Stealth Corvette, Helsingborg, off Gotska, Sandön.

Once an optical structure is modelled this permits optimised liquid crystal cell fabrication, and further modelling for various scenarios, including stealth operational modes. Actual cell experimental data obtained from reflectivity monitoring is compared with theoretical modelling, permitting further LC cell design optimisation. Comparison of theoretical predictions with experimental data as a function of voltage provides a realistic estimation of real contrast variation for potential UAV or maritime platform skins covered with pixelated LC cells.

The electromagnetic response of an optical system can be calculated numerically, using the scattering matrix approach, a convenient method for determining the dispersion relations of guided and surface waves, as stated. A schematic of the simplest case, with a single interface between two adjacent media is described simultaneously by their electrical permittivities $\left(\varepsilon_{1,2}\right)$ and magnetic permeabilities $\left(\mu_{1,2}\right)$ which are functions of frequency and often complex, as shown in figure 4.

The elements of the system's scattering matrix are the well-known Fresnel coefficients in transmission and reflection, and the matrix equation relating the field amplitudes is:

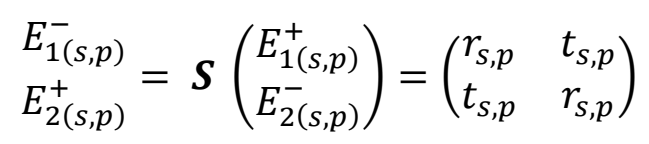

where $E_{1(s, p)}^{+}$and $E_{1(s, p)}^{-}$are the forward and backward travelling electric field amplitudes (in the zdirection) for incident s-polarised or p-polarised (TE and TM mode light respectively) in the ith medium. $\boldsymbol{S}$ is the scattering matrix, whilst $r_{s, p}$ and $t_{s, p}$ are the Fresnel reflection and transmission amplitudes at the interface. The FORTRAN scattering modelling method can accommodate 97 layers, with uniaxial layer characteristics.

\section{Experimental Arrangement and Results}

\subsection{SPR Experimental Arrangement}

The Kretschmann-Raether experimental configuration (figure 6) records reflectivity from fabricated LC cells [15], but the Otto configuration with an air gap between the device surface layer may also be used in cell characterisation [16]. 


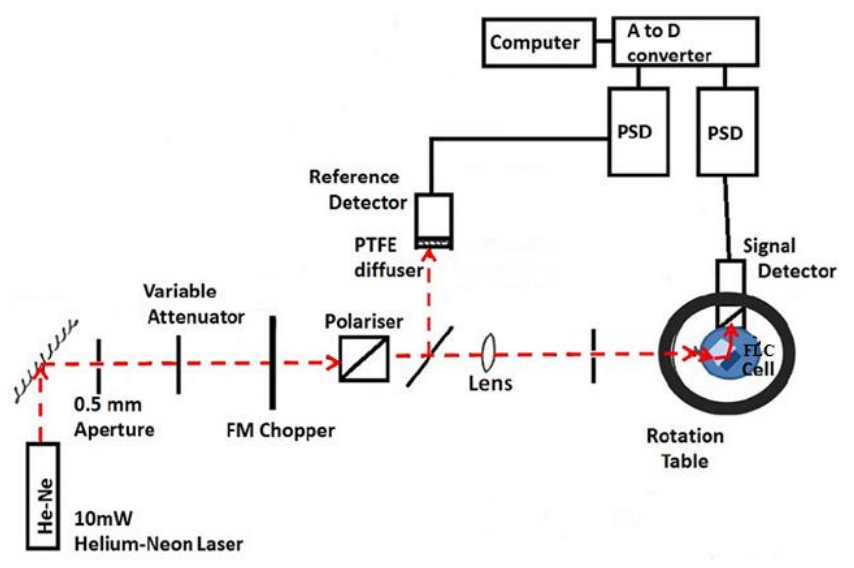

Figure 6 Optical reflectivity Kraetschmann-Raether experimental arrangement.

Cell fabrication is achieved by deposition of $40 \mathrm{~nm}$ Indium Tin Oxide (ITO), with a sputter deposition rate of $10 \mathrm{~nm} \mathrm{~min} \mathrm{~m}^{-1}$. A polyimide layer is then spun onto substrate glass (E06 Soveril) leaving a polymer layer approximately $50 \mathrm{~nm}$ thick. The polyimide is mechanically rubbed unidirectionally several times and cured to achieve low surface tilt alignment of the Ferroelectric Liquid Crystal (FLC) in question. The sample cell has glass surfaces displaced $4 \mathrm{~mm}$ to expose electrode regions for voltage work, and cell surfaces are spaced with 3.5 micron thick mylar, characterised with our reflectivity method. The sample was enclosed in a temperature controlled oven $\left( \pm 0.1^{\circ} \mathrm{C}\right)$ and heated until above the nematic to isotropic phase transition prior to filling. Temperature stabilisation typically took the order of an hour. Filling was achieved by capillary action from the bottom of the assembled cell to prevent creation of air bubbles that interfere with formation of a uniform liquid crystal structure. The cell is placed in a temperature-controlled housing, mounted on a rotating stage along the optical bench. The liquid crystal parameters used in this paper were from a characterised Smectic C* cell of SCE8 from Clariant, (figure 7), with the following phase transitions: SC* $60{ }^{\circ} \mathrm{C} \mathrm{SA} 80{ }^{\circ} \mathrm{C} \mathrm{N} * 102{ }^{\circ} \mathrm{C} \mathrm{I}$. The spontaneous polarisation and rotational viscosity of the SCE8 FLC, measured at $25^{\circ} \mathrm{C}$, are $-4.5 \mathrm{nC} / \mathrm{cm}^{2}$ and $76 \mathrm{mPa}$, respectively (data provided by Clariant). Alignment of SCE8 molecules for parallel aligned surfaces is primarily in the plane of the surface, twisting out quickly in the plane to a fixed angle dependent on cell thickness [17]. We also characterised the visible and near infra-red wavelength response of typical FLC cells [18]. Determination of the optical dielectric constants and deformation effects, after surface treatment of a polyimide alignment layer used within a ferroelectric liquid crystal has also been extensively covered in previous work using surface plasmon resonance [19]. 


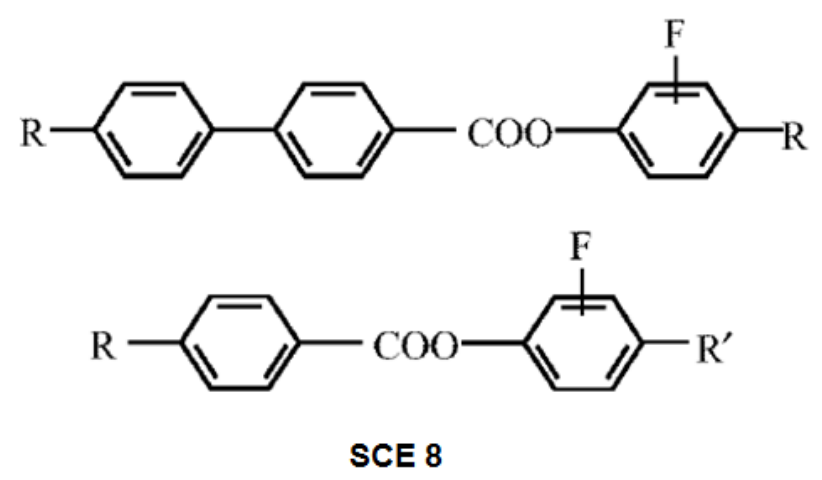

$\mathrm{SmC}^{*}-59^{\circ} \mathrm{C}-\mathrm{SmA}-79^{\circ} \mathrm{C}-\mathrm{N}^{*}-100^{\circ} \mathrm{C}-\mathrm{I}$

Figure 7 SCE 8 FLC.

An available Helium Neon (He-Ne) laser operating at $632.8 \mathrm{~nm}$ provided coupling to the FLC cell guided modes, stepped in angle under computer control, and is a key wavelength for consideration of dawn/dusk stealth platform operation, being scattered less by the atmosphere and battlefield obscurants under those conditions than other wavelengths. Other non-laser visible wavelengths were examined to determine SCE8 and other liquid crystal wavelength dispersive characteristics [18], as well as under an applied electric field [20]. A Frequency Modulated (FM) chopper permits signal and reference with Phase Sensitive Detection (PSD) using lock-in amplifiers to minimise noise (figure 6).

Experimental reflectivity data acquisition takes place with a National Instruments USB 6210. A LabView program controls a motorised rotation stage, recording diode reflectivities from sample / reference beams. A $\theta$ prism movement gives a $2 \theta$ diode turn, ensuring reflected beams strike a diode. As the stage rotates we record reflectivity data against steps. Cells are examined with the Krestchmann configuration (figure 8). Experimental SCE8 liquid crystal cell data under applied voltage: $0-12 \mathrm{~V}$, recorded at $632.8 \mathrm{~nm}$, is shown (figure 9). The recorded angle range illustrated was chosen as it covers the 'direct-reflective' threat angular regime, including 'glint', critical for most platform detection, and bounded by experimental limits on permissible coupling angles. Little useful structural information is obtained below the external critical angle of $14^{\circ}$ at the onset of total internal reflection.

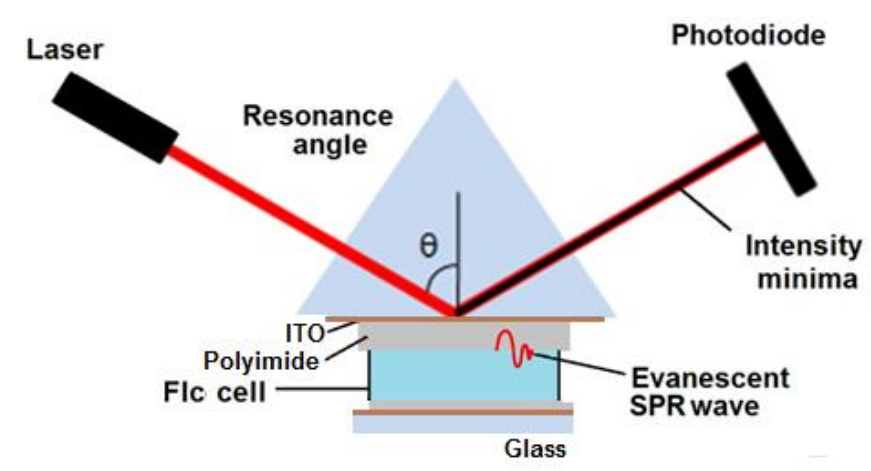

Figure 8 Typical FLC cell showing surface mode excitation. 


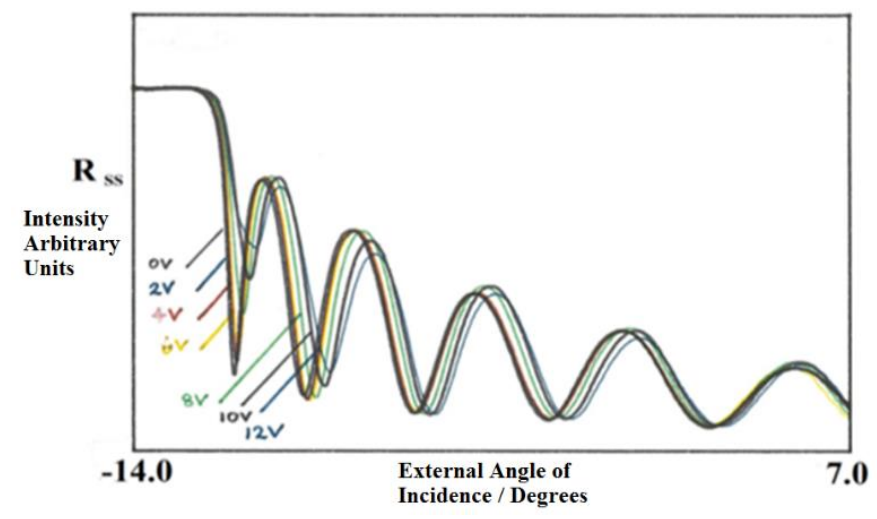

Figure 9 Experimentally recorded reflectivities Rss (s-polarised incident light in, spolarised light out) as a function of applied DC Voltage and externally incident angle in degrees for the Ferroelectric Liquid Crystal SCE8 at room temperature. Intensity is given in arbitrary units prior to fitting against absolute Fresnel theory values.

\section{Optical Predictions and Discussion}

Figure 9 shows the resulting experimental cell reflectivity changes as a function of applied DC voltage. Whilst AC stabilisation FLC cell characteristics have been examined extensively by other workers [21]. Such experimentally obtained reflectivity values allow us to predict the likely achievable adaptive contrast variation at different wavelengths which are most likely facilitate optical detection. Once corrected for Fresnel reflections at the glass surfaces and for internal glass interface angles, experimental data may be compared with theoretical predictions for the modelled in-plane and out-of-plane liquid crystal optical director configuration to achieve extremely good data fits, figure 10 .

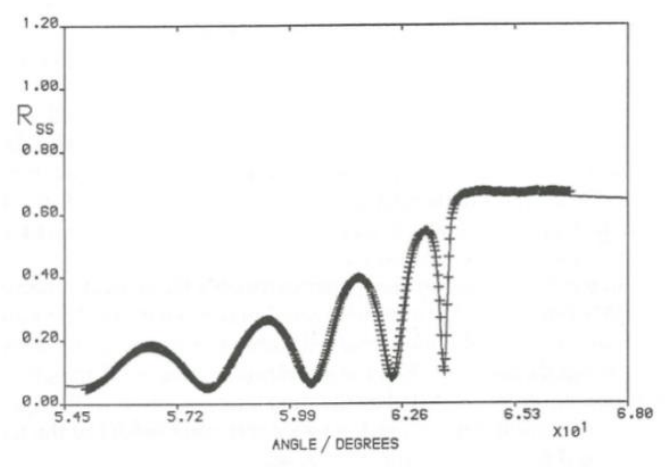

Figure 10 Comparison of experimental data (crosses) with a theoretically predicted reflectivity curve (continuous line) for incident TE polarised light taken from a 3.5 micron parallel aligned SCE $8 \mathrm{FLC}$ cell in the $\mathrm{Sc}^{*}$ phase at $39 \mathrm{C}$.

Using best fit parameters absolute reflectivity values for differing applied cell voltages are obtained. These reflectivity values may then be used to generate a simulated test grid. A pixel test grid shows the possible minimum / maximum contrast and variation for different reflectivity values: minimum black chequers, maximum white chequer, the chosen voltage value provides the other alternating grid chequer (figure $11.0 \mathrm{~V}$ applied), figure $12.6 \mathrm{~V}$ applied, and figure $13.12 \mathrm{~V}$ applied with images generated in Matlab. 


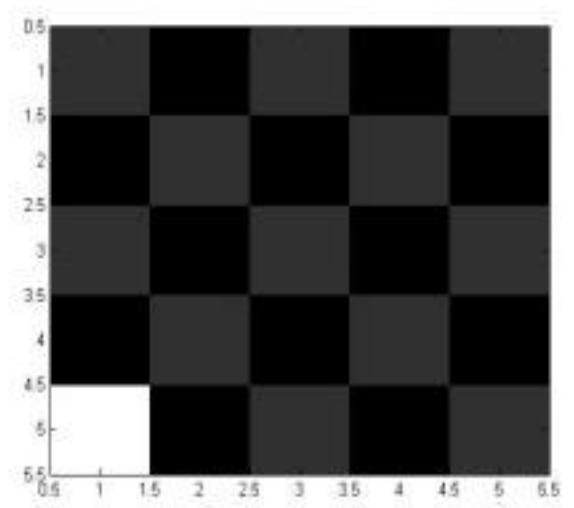

Figure 11 Matlab simulated pixellated test grid with Zero 0 V Applied DC Voltage.

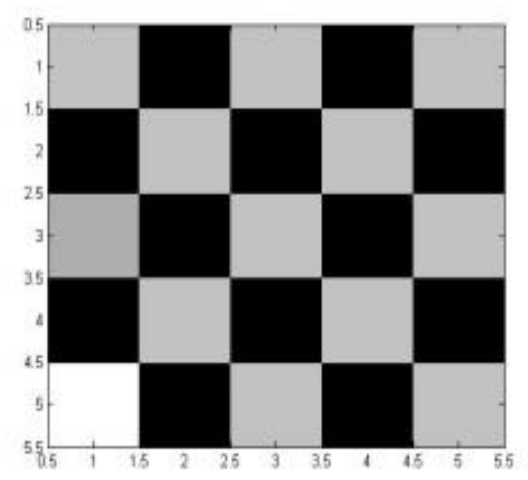

Figure 12 Matlab simulated pixellated test grid with $6 \mathrm{~V}$ Applied DC Voltage.

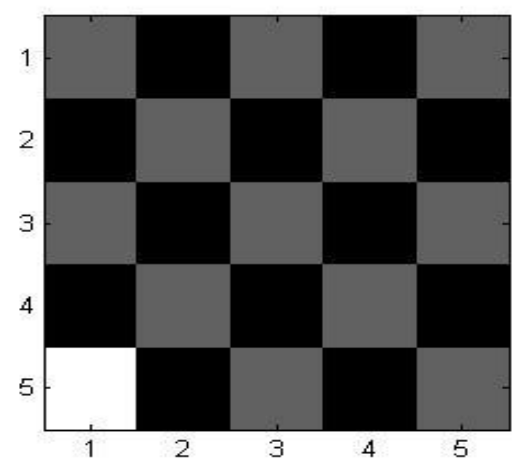

Figure 13 Matlab simulated pixellated test grid with $12 \mathrm{~V}$ Applied DC Voltage.

To model the likely contrast changes possible for a real platform we took a silhouette for the Visby Stealth corvette; we viewed the model in broadside looking sideways towards the platform, a common missile threat attack scenario. Optical values were entered into Matlab in an overall space of almost 60,000 pixel elements, with 64 levels of brightness (0 to 63), although we have used Matrices with up to 120,000 elements [1]. The image space maximised contrast, with some necessary manual edge of platform pixel editing, with the ' 0 ' level corresponding to most of the Visby silhouette, and the ' 63 ' level for the background, combining both elements of sky and sea, as shown in figure 14. 


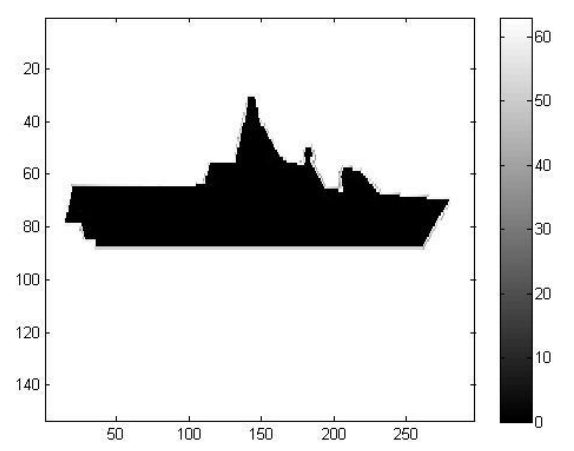

Figure 14 Maximum contrast Visby silhouette.

Normalised contrast may be calculated for target Intensity $I_{T}$ against background $I_{B} \quad u s i n g$ the following equation:

$$
C=\frac{I_{T}-I_{B}}{I_{T}-I_{B}}
$$

which for the initial ship outline conditions provides a maximum contrast of 1 . This approach has been used successfully elsewhere to quantify specific space-based earth imaging assessments of before and after imagery assessment, using a combination of visible and near infra red imagery taking atmospheric absorption and scattering contributions into account $[22,23]$.

Simulated platform reflectivity elements at $632.8 \mathrm{~nm}$ show nearly the maximum silhouette contrast conditions for $0 \mathrm{~V}$ applied, and $12 \mathrm{~V}$ applied, which are both given in figure 15, and figure 16 respectively, with an intermediate applied voltage value (figure 17). It is seen that figure 15 closely matches figure 14 in terms of overall platform contrast, whilst figure 16 with $12 \mathrm{~V}$ applied, has simulated a much reduced contrast against its background, making it much harder to 'see' the platform as a distinct object against its background. For future research a disrupted pattern will likely have a greater impact on platform visibility / invisibility. Figure 17, unsurprisingly is observed to have an intermediate output optical performance when compared with figures 15 and 16 respectively.

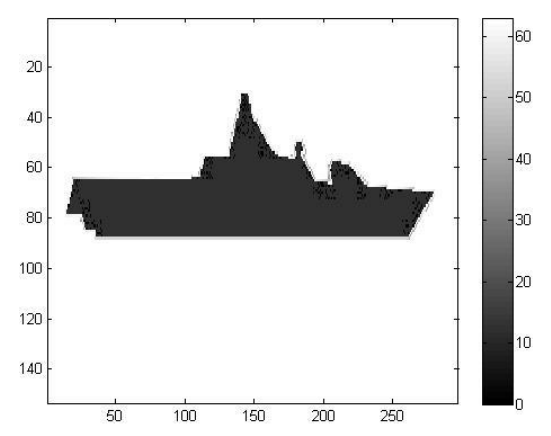

Figure 15 Matlab simulation $0 \mathrm{~V}$ applied. 


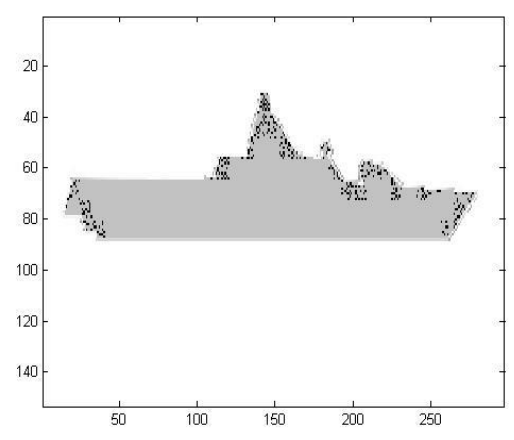

Figure 16 Matlab simulation $12 \mathrm{~V}$ applied.

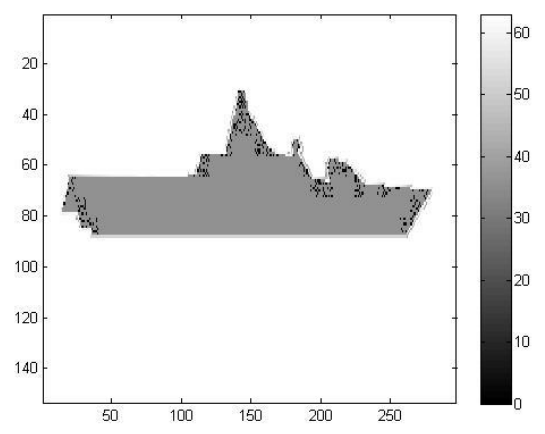

Figure 17 intermediate applied potential difference.

\section{Conclusions}

We have investigated the proof-of-concept validity of using liquid crystal display technology and current readily available liquid crystal materials to achieve low cost platform optical ship stealth in the same manner as in our previous optical UAV platform optical stealth considerations [1]. Although, a simple concept in theory the practical realisation of this in display terms has previously been limited in terms of projection methods and lack of sufficient platform pixelation, limited by power requirements. Modelling and subsequent visualisation with Matlab of a prospective ship platform, coated with a pixelated surface composed of a large number of liquid crystal cells shows large scale changes in overall contrast are possible with potentially low voltage power supply systems, relying upon adaptive incident light illumination manipulation. Small ship platforms, with other low cross sections, themselves carrying smaller UAVs camouflaged with liquid crystal panels in the range $0-12 \mathrm{~V}$ applied voltage, may provide significant contrast changes closely approaching theoretical maximum / minimum contrast conditions. There is little point in applying voltages above $12 \mathrm{~V}$ for two reasons, namely: there is no obvious further benefit in platform reflectivity modification achieved, and secondly the main point of using low voltage passive liquid crystal display materials is to reduce the overall system power consumption burden. An increase in applied voltage will increase power consumption as the square of the applied voltage and so this is a counter-productive strategy for no stealth gain. Future work between the Dartmouth Centre for Seapower and Strategy, with the Changing Character of Warfare Centre, Pembroke, Oxford will look at emerging technologies, with respect to the potential afforded by new synthetic electro-optical materials with temperature, angle, voltage and wavelength 
dependent effects. When combined with disruptive contrast 'patch' applications stealthy ship platforms will provide transformational capabilities. We will also look at how this will influence interactions and dynamics between human actors, the spatial and time domain, and any ethical and moral limitations, which such isoluminant approaches may introduce.

\section{Author Contributions}

The author did all the research work of this study.

\section{Competing Interests}

The authors have declared that no competing interests exist.

\section{References}

1. Lavers $C R$, Johnson R. Experimental modelling with theoretical validation of liquid crystal display elements for UAV optimal (optical) stealth. Proc. SPIE 11525, SPIE Future Sensing Technologies. 2020: 115252U. DOI: 10.1117/12.2585165.

2. Lavers CR. Reeds vol 14: Stealth warship technology. Chinese ed. Beijing: Electronic Industry Press; 2014.

3. Jewish M, Sweetman B. Hide and Seek. Janes Inter Def Rev. 1997; 30: 29-32.

4. Ko DY, Sambles JR. Scattering matrix method for propagation of radiation in stratified media: Attenuated total reflection studies of liquid crystals. J Opt Soc Am A. 1988; 5, 1863-1866.

5. Berreman DW, Scheffer TJ. Bragg reflection of light from single-domain cholesteric liquidcrystal films. Phys Rev Lett. 1970; 25: 902.

6. Azzam RM, Bashara NM, Burns DT. Ellipsometry and polarised light: North Holland, Amsterdam, 1987 (ISBN 0-444-87016-4). xvii + 539 pp. Price Dfl. 75.00. Anal Chim Acta. 1987; 199: 283-284.

7. Thorén O. ADAPTIV - Cloak of Invisibility. BAE Systems. Available from: https://www.baesystems.com/en/feature/adativ-cloak-of-invisibility.

8. Salihoglu O, Uzlu HB, Yakar O, Aas S, Balci O, Kakenov N, et al. Graphene-based adaptive thermal camouflage. Nano Lett. 2018; 18: 4541-4548.

9. $\mathrm{Xu} \mathrm{CY}$, Stiubianu GT, Gorodetsky AA. Adaptive infrared-reflecting systems inspired by cephalopods. Science. 2018; 359: 1495-1500.

10. Sen P. More than meets the Eye. Janes Navy Int. 2002; 107: 003.

11. NASA's Jet Propulsion Laboratory. Adaptive Camouflage [Internet]. 2000. Available from: https://www.techbriefs.com/component/content/article/tb/pub/techbriefs/electronics-andcomputers/1771.

12. Tachi S. Telexistence and retro-reflective projection technology (RPT). Proceedings of the 5th Virtual Reality International Conference(VRIC); 2003 May 13-18; Laval Virtual, France.

13. Highfield R. Now you see him, now you don't - But why? Sunday Telegraph (United Kingdom). 2002. p36.

14. Yablonovitch E. Electrochromic adaptive infrared camouflage. Final report. LA: U.S. Army Research Office; 2005; ADA442555. 
15. Kretschmann $E$, Raether $H$. Radiative decay of non radiative surface plasmon excited by light. Z Naturforsch A. 1968; 23: 2135-2136.

16. Otto A. Excitation of nonradiative surface plasma waves in silver by method of frustrated total reflection. Z Phys . 1968; 216: 398-410.

17. Lavers CR, Sambles JR, Raynes EP, Cann PS. A preliminary investigation of the anomalous out-ofplane tilt alignment in a half-twist ferroelectric liquid crystal cell using the prism-coupling technique. Liq Cryst. 1993; 15: 749-755.

18. Lavers CR, Sambles JR. Wavelength characterisation of ferroelectric liquid crystal cells. Jpn J Appl Phys. 1991; 30: 729.

19. Lavers CR. Determination of the optical dielectric constants, and deformational effects, after surface treatment, of a polyimide alignment layer used within a ferroelectric liquid crystal device system. Thin Solid Films; 1996; 289: 133-139.

20. Lavers CR, Cann PS, Sambles JR, Raynes EP. Optical mode characterisation of the configuration of a thin ferroelectric liquid crystal cell under an applied electric field. J Mod Opt. 1991; 38: 14511461.

21. Elston SJ, Sambles JR, Clark MG. The mechanism of ac stabilization in ferroelectric liquidcrystal-filled cells. J Appl Phys. 1990; 68: 1242-1246.

22. Lavers CR, Mason TR. High-resolution IKONOS satellite imagery for normalized difference vegetative index-related assessment applied to land clearance studies. J Appl Remote Sens. 2017; 11, 035008.

23. Lavers CR, Mason T. Ikonos Satellite imagery for NDVI related assessment applied to land clearance studies. Proceedings Volume 11534, Earth Resources and Environmental Remote Sensing/GIS Applications XI. 2020 September 23; online. DOI: 10.1117/12.2584921.

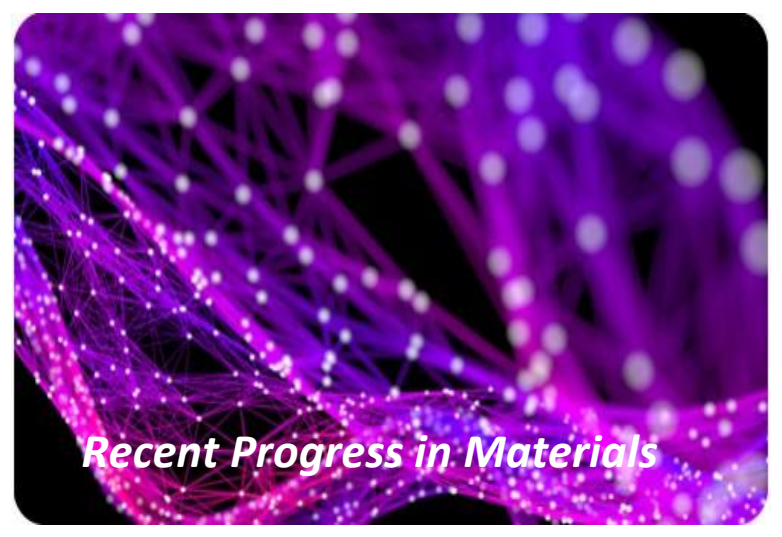

Enjoy Recent Progress in Materials by:

1. Submitting a manuscript

2. Joining in volunteer reviewer bank

3. Joining Editorial Board

4. Guest editing a special issue

For more details, please visit:

http://www.lidsen.com/journals/rpm 